\title{
High impact of in situ dextran coating on biocompatibility, stability and magnetic properties of iron oxide nanoparticles
}

\author{
Z. Shaterabadi ${ }^{1}$, G.Nabiyouni ${ }^{1 *}$, M. Soleymani ${ }^{2}$ \\ 1. Department of Physics, Arak University, Arak, 38156-88349, Iran \\ 2. Department of Chemical Engineering, Arak University, Arak, 38156-88349, Iran
}

\begin{abstract}
Biocompatible ferrofluids based on dextran coated iron oxide nanoparticles were fabricated by conventional co-precipitation method. The experimental results show that the presence of dextran in reaction medium not only causes to the appearance of superparamagnetic behavior but also results in significant suppression in saturation magnetization of dextran coated samples. These results can be attributed to size reduction originated from the role of dextran as a surfactant. Moreover, weight ratio of dextran to magnetic nanoparticles has a remarkable influence on size and magnetic properties of nanoparticles, so that the sample prepared with a higher weight ratio of dextran to nanoparticles has the smaller size and saturation magnetization compare with the other samples. In addition, the ferrofluids contain such nanoparticles have an excellent stability at physiological $\mathrm{pH}$ for several months. Furthermore, the biocompatibility studies reveal that surface modification of nanoparticles by dextran dramatically decreases the cytotoxicity of bare nanoparticles and consequently improves their potential application for diagnostic and therapeutic purposes.
\end{abstract}

Keywords: Iron oxide magnetic nanoparticles, Co-precipitation, Dextran coating, Magnetic properties, Superparamagnetism, Biocompatibility

\footnotetext{
*Corresponding author. E-mail address: g-nabiyouni@araku.ac.ir
} 


\section{Introduction}

In recent years, magnetic nanoparticles (MNPs) have been widely attracted a great deal of attention in nanomedicine due to their sizes, comparable to biological molecules [1]. However, among various metal or metal oxide MNPs, only a few of them have reached preclinical or clinical trials, mainly because of their potential toxicity and rapid oxidation [2]. Among them, iron oxide nanoparticles (NPs), especially magnetite $\left(\mathrm{Fe}_{3} \mathrm{O}_{4}\right)$, are of growing interest for researchers owning to their superior magnetic properties as well as high degree of biocompatibility and safety [3]. Magnetite lies in the category of inverse spinel ferrites with the ferrimagnetic behaviour [4]. In such structure, oxygen ions form a face centred cubic configuration in which $\mathrm{Fe}^{3+}$ ions occupy tetrahedral and octahedral sites equally, and $\mathrm{Fe}^{2+}$ ions locate in the octahedral sites. Although a ferromagnetic spin coupling between $\mathrm{Fe}^{3+}$ and $\mathrm{Fe}^{2+}$ ions takes place in the octahedral sites, an antiparallel spin coupling between moments in octahedral and tetrahedral sites is responsible for ferrimagnetic behavior in magnetite $[5,6]$.

The magnetic properties of MNPs may be either modified or even deteriorated by size reduction, even though they are influenced by various physicochemical and synthesis parameters. In nanometer scales, because of signifying the role of surface region atoms in determining magnetic properties of the whole system, new and in some case unusual magnetic properties appears in MNPs. These anomalous magnetic properties, include a reduced saturation magnetization [7, 8], shifted hysteresis loop [7, 9], open hysteresis loop (high field irreversibility) [9, 10] and the nonsaturated magnetization in high fields $[11,12]$. Recently, these properties have been explained based on the core-shell morphology of fine MNPs consist of ferri- or ferromagnetic alignment of core spins surrounded by a spin glass-like surface layer [7, 13]. However, one of the most intriguing properties of ferri- or ferromagnetic nanoparticles is the appearance of superparamagnetic behavior in the regions smaller than the magnetic domains. In fact, superparamgnetism is a transition of ferri- or ferromagnetic state towards paramagnetic-like behavior with high susceptibility and saturation magnetization [5]. Although the tendency of losing magnetization completely after removing field, appearing in superparamagnetic nanoparticles, is a disadvantage in some applications of MNPs such as magnetic recording media [14], this property could be a particular privilege in ferrofluids used in biomedical applications.

In fact, fluctuations of magnetization in easy directions minimize the magnetic interactions 
between NPs in colloidal suspension and consequently by improving dispersibility and chemical stability of nanoparticles, reduce the risk of embolism in blood vessels [6, 15-17]. For this reason, recently, ferrofluids based on superparamagnetic iron oxide NPs have been used as one of the most widely agents in theranostic (therapeutic and diagnostic) applications such as magnetic fluid hyperthermia [18-20], targeted drug delivery [21, 22], magnetic resonance imaging (MRI) [23-27], gene therapy [28], magnetic particle imaging [29], cell labelling [30], immunoassay [31] and DNA detection [32].

The hydrodynamic size and surface physicochemical properties of MNPs are two important factors determining the blood circulation time within the body and their biological fate in biomedical applications [27, 33-35]. Hence, in order to increase the efficiency of superparamagnetic iron oxide NPs in desired applications, especial surface coatings are usually required. Dextran is a natural polymer with some clinical applications used as an antithrombotic for reducing blood viscosity [34]. It is also non-toxic, water soluble, and also has a long blood circulation time and high bio-affinity [24, 27, 36-40]. Dextran is currently one of the most common coatings utilized in various clinical trials.

In recent years, a large number of published works have been devoted to the theoretical and experimental investigation of magnetic properties of MNPs modified by size reduction. On the other hand, biomedical applications (diagnostic and therapeutic) of core-shell MNPs, containing few nanometer-sized magnetic cores, have been one of the most important forefronts in nanoscience researches. Hence, it seems, there is a great need for a systematic study of size shrinkage effects in MNPs utilized in theranostic applications in order to achieve the more control over the magnetic properties, biocompatibility, quality and reproducibility of samples.

In this study, bare and dextran coated iron oxide NPs were successfully synthesized by a facile and one-step co-precipitation method. Morphology and size distribution of MNPs were determined by field emission scanning electron microscopy (FESEM) and transmission electron microscopy. Hydrodynamic size was measured by dynamic light scattering (DLS). Crystal structure and surface coating characteristics were investigated by X-ray diffraction (XRD), Fourier transform infrared spectroscopy (FT-IR), and thermogravimetric analyses (TGA). The effect of dextran coating on the magnetic properties of bare MNPs was investigated by VSM analysis. Furthermore, the biocompatibility of obtained samples was evaluated by MTT assay. In addition to the investigation of magnetic properties of iron oxide NPs modified by in situ dextran 
coating, an especial emphasis is given to reduce the toxicity and improve the viability of dextran coated NPs for biomedicine and bioengineering applications.

\section{Materials and methods}

\subsection{Materials}

Iron (III) chloride hexahydrate $\left(\mathrm{Fe} \mathrm{Cl}_{3} \cdot 6 \mathrm{H}_{2} \mathrm{O}\right)$, iron (II) chloride tetrahydrate $\left(\mathrm{Fe}_{2} \cdot 4 \mathrm{H}_{2} \mathrm{O}\right)$ and Sodium hydroxide $(\mathrm{NaOH})$ were purchase from Merck company. $40 \mathrm{kDa}$ dextran $\left(\mathrm{H}\left(\mathrm{C}_{6} \mathrm{H}_{10} \mathrm{O}_{5}\right)_{x} \mathrm{OH}\right)$ was obtained from Sigma Aldrich company. All chemicals were used as received, without any further purification.

\subsection{Synthesis of bare and dextran coated magnetite NPs}

Bare and dextran coated magnetite nanoparticles were synthesized through co-precipitation of ferrous and ferric salts with molar ratio of 1:2 in alkaline solution. As schematically illustrated in Fig. 1, $0.250 \mathrm{~g}$ of $\mathrm{Fe} \mathrm{Cl}_{2} \cdot 4 \mathrm{H}_{2} \mathrm{O}$ and $0.665 \mathrm{~g}$ of $\mathrm{Fe} \mathrm{Cl}_{3} \cdot 6 \mathrm{H}_{2} \mathrm{O}$ were dissolved in $100 \mathrm{ml}$ distilled water under nitrogen atmosphere and vigorous stirring at $80^{\circ} \mathrm{C}$ in a three-neck flask equipped with condenser. $12 \mathrm{ml}$ of $1 \mathrm{M}$ aqueous solution of sodium hydroxide (as a reducing agent) was then added to adjust the solution $\mathrm{pH}$ to 12 . Finally, the reaction was continued for $1 \mathrm{~h}$ under nitrogen protection. In order to prepare the dextran coated NPs, two different weight ratios of dextran to magnetite NPs (i.e. 1:1 and 2:1) were dissolved in reaction medium prior to adding sodium hydroxide. The resultant black precipitate was then centrifuged and washed for three times with deionized water. The large aggregates were then removed by centrifugation at 1000 rpm. The sample prepared with weight ratio of dextran to MNPs 1:1 was named as sample A, and that prepared by weight ratio of 2:1 as sample B.

\subsection{Characterization}

The crystal structure of samples was studied using $\mathrm{X}^{\prime}$ Pert Pro X-ray diffractometer with $\mathrm{Cu}-$ $K_{\alpha}$ radiation. FTIR spectroscopy analysis was performed on Bruker Vertex 70 spectrometer 
(with resolution of $0.5 \mathrm{~cm}^{-1}$ ). The changes of residual mass of pure dextran and the MNPs with temperature were characterized by TG analysis and using Perkin Elmer instrument. The size distribution and morphology of the bare and dextran coated NPs were investigated by field emission scanning electron microscope (FESEM, Tscan Co., Czech Republic) and also transmission electron microscope (TEM, Phylips-CM120) with an acceleration voltage of 120 $\mathrm{kV}$. The hydrodynamic size of the dextran coated NPs was assessed by dynamic light scattering (DLS, Malvern zetasizer-ZEN3600). The magnetic properties of the bare and dextran coated NPs were studied at the room temperature, using a vibrating sample magnetometer (VSM, Meghnatis Kavir Kashan Co., Iran). Cytotoxicity of samples was evaluated by MTT assay with L929 cell line (mouse fibroblast).

\subsection{Cell viability study through the methyl thiazol tetrazolium bromide (MTT) assay}

In vitro cytotoxicities of the bare and dextran coated magnetite nanoparticles were evaluated using the methyl thiazol tetrazolium bromide (MTT) assay. In summery, the L929 cells with a concentration of $1 \times 10^{5}$ cells per $m l$ were incubated at $37^{\circ} \mathrm{C}$ for $24 \mathrm{~h}$ in a 96-well plate humidified by $5 \% \mathrm{Co}_{2}$ atmosphere. Different concentrations (50, 100, 200, 300, 400 and $500 \mu \mathrm{g} \mathrm{ml}^{-1}$ ) of the bare and dextran coated nanoparticles (sample B) were added to test cells and afterwards, the cells were cultured for another $24 \mathrm{~h}$. in the next step, $15 \mu \mathrm{l}$ of MTT solution was added to each well. Then, cells incubated again for $4 \mathrm{~h}$. Finally, optical density was evaluated at $570 \mathrm{~nm}$. Cell viability was calculated as follows:

$$
\text { Cell viability }(\%)=\left[\left(A_{\text {test }}-A_{\text {blank }}\right) /\left(\mathrm{A}_{\text {control }}-A_{\text {blank }}\right)\right] \times 100 \%
$$

where, $A_{\text {test }}$ is the absorbance of a well containing test cells treated with NPs, $A_{\text {control }}$ is the absorbance of a well containing untreated control cells, and $A_{\text {blank }}$ is the absorbance of a blank well containing medium only.

\section{Result and discussion}

Iron oxide NPs with superparamagnetic property can be synthesized by various wet chemical methods such as co-precipitation [41], microwave [42], sonochemical [43], solvothermal [44], 
hydrothermal [45], etc. Among these methods, co-precipitation is one of the simplest and the most common techniques with large-scale producibility in which the nanoparticle shapes and sizes can be easily modified by tailoring the synthesis parameters [46].

In this research, the process of synthesizing the bare and dextran coated iron oxide NPs was accomplished by co-precipitation method with considering below points:

1- Single phase magnetite is obtained at reaction temperatures above $50^{\circ} \mathrm{C}$ [47]. Moreover, in order to produce of MNPs with high degree of crystallinity (evolution from amorphous to crystalline structure) and also formation of spinel structure, aging in the temperature range of $80^{\circ} \mathrm{C}$ to $100^{\circ} \mathrm{C}$ for at least $1 \mathrm{~h}$ is essential [48-51].

2- Bubbling inert gas through the solution not only prevents magnetite nanoparticles from oxidation and consequently from producing undesirable $\mathrm{Fe}(\mathrm{OH})_{3}$ segments [52] but also reduces their size [53]. Furthermore, inert atmosphere can suppress oxidation of $\mathrm{Fe}^{2+}$ ions at $\mathrm{pH}$ of above 6 and consequently hinder the formation of maghemite phase which reduces magnetic saturation of nanoparticles [50].

3- Above a critical $\mathrm{pH}$ value, the secondary particle growth by Ostwald ripening does not take place under aging in conditions of synthesis and consequently nanoparticle size remains stable over time. This critical value is depended on the ionic strength of reaction solution and usually comes in the range of 11 to $12[54,55]$.

4- Dextran coating is usually performed in the temperature range of $60^{\circ} \mathrm{C}$ to $85^{\circ} \mathrm{C}$ and aging time of 15 to $60 \mathrm{~min}[2,38,56]$, and $\mathrm{pH}$ of above $10[27,38,56]$.

According to above discussion, the precipitation reactions were performed under nitrogen atmosphere at temperature of $80^{\circ} \mathrm{C}$. The required aging time was considered as $1 \mathrm{~h}$ and the suitable value of reaction acidity was regulated to 12 .

\subsection{Field emission scanning electron microscopy}

Field emission scanning electron microscopy (FESEM) of the bare and dextran coated NPs are shown in Fig. 2. As can be seen, size distribution of the bare NPs is broader than that of the dextran coated NPs. Furthermore, average particle size of the bare NPs $(17.8 \mathrm{~nm})$ is greater than 
that of the dextran coated NPs (14 and $10.7 \mathrm{~nm}$ for the samples A and B respectively). This result is probably due to the presence of dextran in reaction medium which inhibits growth of NPs after nucleation through encapsulating of them. In addition, the dextran coated sample B has the smaller size with sharper size distribution compare with the other samples. This outcome is likely because of completely coating of all NPs in the sample B which in turn causes to the more stability of them for several months. Fig. 3 visually compares the aqueous dispersion stability of the bare and dextran coated sample B at physiological pH. The bare NPs immediately after preparing agglomerate and settle in the bottom of the container, whereas, the suspension containing the sample B remains stable for a long time. Moreover, color of the bare NPs gradually changes from back to brown after passing time about one day, indicating the surface oxidation. However, color of the dextran coated NPs remains unchanged over several months, confirming the well coating of particles with dextran.

\subsection{Transmission electron microscopy}

Fig. 4 shows TEM images of the bare and dextran coated NPs of sample B. As can be seen, the bare NPs are completely agglomerated. The NPs of sample B are spherical in shape. They also have a relatively sharp distribution of size around $9 \mathrm{~nm}$ which is measured by averaging the size of NPs in several images. Moreover, they are approximately separated by a thin layer (thickness about $3 \mathrm{~nm}$ ) of polymer covering. The existence of polymer surrounding the magnetic core can be indicative of the attachment of dextran molecules to the surface of NPs.

Dextran is abundant in hydroxyl groups and therefore it can easily attach to the surface of magnetic nanoparticles by hydrogen bonding [34, 36, 57]. Although single hydrogen bonds in dextran are relatively weak, the large number of hydroxyl groups over the length of dextran molecule can guarantee the firmly attachment of dextran to the surface of nanoparticles [33].

It is reported by several research groups that alcoholic hydroxyl groups belonging to the adsorbed dextran on the surface of ferrite nanoparticles reduce the $\mathrm{pH}_{\mathrm{PZC}}(\mathrm{pH}$ at point of zero charge, PZC) from about 7 to $4[36,57,58]$. In fact, dextran coated ferrite nanoparticles possess negative charge under physiological conditions $(\mathrm{pH} \mathrm{7,} \mathrm{and} \mathrm{ionic} \mathrm{strength} 0.15 \mathrm{M} \mathrm{NaCl}$ ) and stabilize trough electrostatic interactions $[24,57,58]$. In addition to van der Waals repulsion 
between coatings, steric repulsive force resulted from long chains of coated polymer is another reason for protecting nanoparticles against agglomeration [59]. The tendency of nanoparticles to adhesion is dominantly originated from strong magnetic dipole-dipole interactions between them. Although the formation of aggregates is depended on the relative orientation of magnetic dipoles, random orientation of magnetic moments due to their Brownian motion cannot prevent nanoparticles from agglomeration. In fact, the bare nanoparticles tend to form long chains in which magnetic moment of each particle aligns with that of the neighbor along the chain [60, $61]$.

\subsection{Dynamic light scattering}

DLS analysis was used to measure the hydrodynamic size of dextran coated NPs of sample B. As can be seen in Fig. 5, this sample has a relatively sharp peak around $69 \mathrm{~nm}$ with a size distribution in the range of 50-100 $\mathrm{nm}$. Moreover, the zero percent measured for the number of particles with size of larger than this range confirms the lack of presence of any aggregates in this sample. In addition, the size measured by this method is much larger than those obtained by TEM and FESEM analyses. Such a result is acceptable considering with the severe dependency of hydrodynamic size to many agents other than core size such as surface structure, water hydration and concentration of any ions in the medium.

In biomedical applications, the hydrodynamic size decides the biological fate of nanoparticles because the bigger particles are quickly taken up by liver or spleen, while the smaller particles are rapidly removed through extravasation and renal clearance. In fact, particles in the range of 10-100 nm elude the reticuloendothelial (RES) system and therefore due to having a longer blood circulating time can offer the more effective distribution in certain tissues [27, 33-35]. Hence, the dextran coated NPs prepared in this research, due to their suitable hydrodynamic size, have an excellent potential for in vivo bioapplications.

\subsection{X ray diffraction}

The XRD patterns of the bare and dextran coated NPs as well as pure dextran are shown in Fig. 6. As can be seen, dextran has a broad peak at $2 \theta \cong 17^{\circ}$ indicating its amorphous structure [2], 
Whereas, sharp peaks of the bare NPs confirm highly crystalline structure of these particles. Also, all reflection peaks in XRD spectrum of the bare nanoparticles are in accordance with JCPDS card (No. 75-0033) associated with cubic phase of $\mathrm{Fe}_{3} \mathrm{O}_{4}$ (space group: $\mathrm{Fd}-3 \mathrm{~m}$ ). However, the presence of dextran due to its amorphous structure and also its effect on size reduction of the samples $A$ and $B$ causes to broaden observed peaks in XRD pattern of the dextran coated NPs [23]. In addition, the average crystallite size (D) is calculated by Scherrer's equation:

$$
D=\frac{0.9 \lambda}{\beta \cos \theta}
$$

which $\lambda$ is the wavelength of the incident X-ray $\left(\lambda_{C u}=1.54 A^{\circ}\right), \beta$ and $\theta$ denote the full-width at half-maximum (FWHM) and diffraction angle of the peak respectively, corresponding to maximum intensity. The average crystallite sizes of the bare NPs and also magnetic core of the samples $\mathrm{A}$ and $\mathrm{B}$ calculated by applying above equation to the highest intensity peak of XRD spectrum at $2 \theta \cong 35.63^{\circ}$ are about $28.42,14.20$ and $10.15 \mathrm{~nm}$ respectively. The crystallite sizes of the dextran coated NPs consist with the average particle sizes shown in the FESEM and TEM images. Nevertheless, there is a significant discrepancy between corresponding values for the bare NPs which can be attributed to broad size distribution of the bare NPs, so that, the average particle sizes for the bare NPs obtained from various FESEM images are different.

\subsection{Fourier transform infrared spectroscopy}

Fig. 7 illustrates the FT- IR spectra of pure dextran as well as the bare and dextran coated NPs. The dextran spectrum exhibits several characteristic absorption bands as follows: The band appeared at $3420 \mathrm{~cm}^{-1}$ is due to the hydroxyl stretching vibration of the polysaccharide, the band at about $2903 \mathrm{~cm}^{-1}$ is assigned to stretching vibration of $-\mathrm{CH}_{2}-$ groups, the band around $1636 \mathrm{~cm}^{-1}$ is characteristic peak of $\mathrm{C}=0$ stretching vibration groups, the band in the region of $1641 \mathrm{~cm}^{-1}$ is owing to water molecule bonding and finally the bands at about 918, 857, and 756 $\mathrm{cm}^{-1}$ are related to a-glucopyranosering deformation modes. These results well agree with which obtained by other research groups [27, 62]. In the spectrum of the bare NPS, the characteristic peaks of $\mathrm{Fe}_{3} \mathrm{O}_{4}$ nanoparticles are occurred at 449.4 and $588.3 \mathrm{~cm}^{-1}$ which are 
attributed to the bending and stretching vibration modes of the $F e-O$ bond, respectively. Also, a broad absorption peak at $3399 \mathrm{~cm}^{-1}$ is related to the stretching vibration mode of - $\mathrm{OH}$ bond [33]. In the dextran coated NPs (especially in sample B), the characteristic peaks of $\mathrm{Fe}_{3} \mathrm{O}_{4}$ are appeared in the same regions with a noticeable slight shift to higher wavenumbers (462.1 and $599.8 \mathrm{~cm}^{-1}$ ). The slight red-shifts in normal stretching and bending modes of the $F e-O$ bonds suggest that the interaction between dextran molecules and $\mathrm{Fe}_{3} \mathrm{O}_{4}$ nanoparticles could help to form a hydrogen bonding between the $\mathrm{Fe}-\mathrm{O}$ bond of the nanoparticles surface and abundant hydroxyl groups of dextran during synthesis process.

\subsection{Thermogravimetric analyses}

TGA curves of the bare and dextran coated NPs as well as pure dextran in the temperature range of $30-1000^{\circ} \mathrm{C}$ under nitrogen atmosphere are shown in Fig. 8. An initial small weight loss around $150^{\circ} \mathrm{C}$ for all samples is due to the evaporation of adsorbed water from their surface which agrees with the other reported results [24, 62]. In case of the pure dextran, polymer decomposition mainly takes place at temperature interval of $180-730^{\circ} \mathrm{C}$, whereas, the TGA curve of the dextran coated NPs shows two stages of degradation: $28.56 \%$ at $181-616^{\circ} \mathrm{C}$ and $19.50 \%$ at $616-894^{\circ} \mathrm{C}$ for sample $\mathrm{A}$ and also $28.58 \%$ at $180-525^{\circ} \mathrm{C}$ and $20.23 \%$ at $525-906^{\circ} \mathrm{C}$ for sample B. The degradation peak in the first step of weight loss for the dextran coated samples is approximately occurred at $270{ }^{\circ} \mathrm{C}$ which is lower than which happens for the pure dextran $\left(307^{\circ} \mathrm{C}\right)$. Such observed shift is expected to be due to catalytic effect of iron [24, 62]. However, degradation rate of dextran in the samples A and B is much slower than that of the pure dextran. Especially, the residual weight for the pure dextran at temperature of $600^{\circ} \mathrm{C}$ is only about $10 \%$ of its total weight, whereas, the same value for the dextran coated samples is more than $40 \%$. The reason for this different behavior of dextran could be due to its firmly binding to magnetite NPs through hydrogen and coordinative bonds [62].

The percentage of dextran molecules attached to the surface of NPs in the samples A and B can be easily calculated via measuring the difference in residual weight between the bare and dextran coated NPs. These results indicate that the coating efficiency of the samples A and B are about $50.12 \%$ and $53.89 \%$ respectively. Hence, the the content of adsorbed dextran on the surface of magnetite NPs is not sensitive to the weight ratio of dextran to NPs. To the best of our 
knowledge, there is no report based on the effect of weight ratio of dextran to MNPs on the coating efficiency. In fact, it is reported that the geometry of dextran chains determines the efficiency of polymer coating. On the other word, the heavier dextran has the more chance to attach to MNPs due to long branches of its molecules. Therefore, coating efficiency increases with the molecular weight of dextran [27].

Although there is a little discrepancy between coting efficiency of the samples A and B, aqueous dispersion stability of the sample B is much better than the other. The sample B is a perfect ferroflouid with high degree of stability in which any aggregation isn't seen after ultracentrifugation. However, this result doesn't mean that the sample A is unstable. In fact, the sample A has an acceptable stability at physiological pH. Nevertheless, some aggregates seen after centrifuging the sample A at low speed is probably due to two main reasons including unsuccessful coating of some NPs as well as the existence of some NPs with bigger size. In this research, according to FESEM and TEM results, the sample A has the broader size distribution compared to the sample B. This outcome can offer an evidence for this fact that the weight ratio of 1:1 for dextran to magnetite NPs is not enough in order to surface modification of all NPs. This result is consistent with other published work [27].

\subsection{Magnetic properties}

In order to investigate magnetic properties of the bare and dextran coated magnetite NPs, the variations of magnetization as a function of applied magnetic field (hysteresis loops) at room temperature were studied. The results are presented in Fig. 9 and summarized in Table 1. As can be seen, coercivity and remanence of the dextran coated NPs are nearly zero, indicating the superparmagnetic behavior of these samples. However, saturation magnetization in these samples dramatically diminishes compare with the bare NPs.

One of the simplest and the most successful models in understanding magnetic phenomena in single domain particles is the Stoner-Wolfforth model. According to this model, in the absence

of magnetic field, magnetization is stable and orients parallel to one of easy directions [63]. The easy directions (parallel and anti-parallel with each other), determined by magnetic anisotropy of 
system, are energetically degenerated and separated by an anisotropy energy barrier $\left(E_{A}\right)$ expressed by following equation $[64,65]$.

$$
E_{A}=K V \sin ^{2} \theta
$$

which, $\mathrm{K}$ is the magnetic anisotropy constant, $\mathrm{V}$ is the volume of the particles and $\theta$ is the angle between the direction of magnetization and the easy axis of magnetization. The magnetic anisotropy includes two distinct parts: magnetocrystalline and shape anisotropy which are respectively inherent property and dependent on the shape of particles [66].

Application of external magnetic field, by removing the energy degeneracy and consequently decreasing energy barrier in one direction and increasing it in another, favours one of these directions (with lower energy) and therefore magnetization can be saturated in the field direction $[65,67]$. However, in ultrafine NPs, the volume of particles is small enough that thermal energy $K_{B} T$ overcomes to the energy barrier $\left(E_{A}\right)$. Thus magnetization of each particle is no longer stable and fluctuates rapidly, resulting in disappearing of coercivity and remanence. Such a this system is known as superparamagnetic [5, 65, 67].

Although the magnetocrytalline anisotropy for magnetite is reported in the range of $11-14 \frac{\mathrm{KJ}}{\mathrm{m}^{3}}$ [68], because of variety in the particle shape and thereby uncertainty in determination of shape anisotropy [66], there is a broad distribution of effective magnetic anisotropy from 10 to $41 \frac{\mathrm{KJ}}{\mathrm{m}^{3}}$ in the literature [69-71]. Nevertheless, the reported superparamagnetic limit for magnetite NPs is reported around $15 \mathrm{~nm}$ [72]. In this research, according to FESEM and TEM images, average particle sizes of the samples A and B are found to be about 14 and $9 \mathrm{~nm}$ respectively, which are in agreement with the mentioned limit for appearance of superparamagnetic behavior.

Another interest result obtained by magnetic measurements is decrease in saturation magnetization $\left(M_{S}\right)$ of the bare and dextran coated NPs compare with the magnetite bulk magnetization which is reported to be in the range of 84- $92 \mathrm{emu} / \mathrm{g}[5,8]$. This decrease in saturation magnetization is more significant in the dextran coated NPs, so that the $M_{S}$ value of the sample B is nearly eight times smaller than that of the bare NPs.

The reported magnetization values in the Table 1 are measured in terms of whole material weight, while based on TGA measurements, only about $43.84 \%$ and $40.07 \%$ of weight of the samples A and B consist of magnetic material which can participate in magnetization of whole system. Therefore, a remarkable portion of drastic suppression in magnetization of the dextran 
coated NPs can be attributed to the presence of non-magnetic dextran shell in such samples. According to a simple calculation with consideration coating efficiency about $50.12 \%$ and $53.89 \%$ for the samples $\mathrm{A}$ and $\mathrm{B}$, the magnetization values for samples $\mathrm{A}$ and $\mathrm{B}$ are estimated to be about 27.49 and $25.12 \mathrm{emu} / \mathrm{g}$ respectively. Since our estimated values are much higher than those given in Table 1, there must be something else lying behind. A possible explanation is as follow:

According to the core-shell model, MNPs consist of a core with ferri- or ferromagnetically aligned spins surrounded by a spin disordered shell usually named magnetic dead layer [7, 13]. Although surface spins are completely disordered, this situation is really different with random configuration of spins in paramagnetic phase $[7,9,13]$. In fact, since surface spins are coupled with core spins via exchange interactions, surface spin orientations are occurred in specific angles with respect to core spins which is called canting angles. In the other words, canted spins in surface layer have multiple configurations for each orientation of the core magnetization [7, 9].

The elimination of magnetic order in the surface layer could be originated from the lack of longrange crystalline order in this region [13]. In fact, due to structural distortions, magnetic cations in the surface layer possess less and variable coordination, resulting in perturbation in crystal field and consequently modification of magnetocrystalline anisotropy in this region which is called surface anisotropy [12]. In the other words, canted spins are created by breaking atomic bonds and therefore frustrating of exchange interactions in the surface layer [10-12].

Although surface anisotropy as well as broken atomic bonds can satisfactorily confirm the existence of magnetically disordered shell, these are not sufficient for explanation of shell thickness [7, 73]. The numerical calculations, taking the above effects into account, indicate that shell is ultra-thin with a thickness about one or two atomic layers [9, 73, 74], whereas, the experimentally determined thickness is comparable to or greater than the lattice constant [7]. For example, Kim et.al reported that surface layer for magnetite nanoparticles extends to a thickness of twice its lattice parameter which is about $0.832 \mathrm{~nm}$ [8]. This discrepancy is explained by an extra anisotropy originated from distribution of exchange fields [7]: Near the surface, the electrostatic potential or Madelung potential is different from that of inside the core because there are no ion outside the crystal that contributes to the Madelung potential. Hence, under consideration the portion of Madelung potential in determining exchange interactions, a 
distribution of exchange fields and therefore magnetic disorder occurs. Owning to the long-range nature of Coulomb interactions present in Madelung potential, this effect diminishes only gradually with increasing distance from the surface. Therefore, inclined surface spins gently align with the ferri- or ferromagnetically ordered spins of core.

Assuming that, the shell thickness ( $t$ ) is independent of nanoparticle diameter (D) and net magnetization of the shell is nearly zero, the variation of saturation magnetization $\left(M_{s}\right)$ with $\mathrm{D}$ will then be described by following equation where $M_{s 0}$ is the $M_{S}$ of bulk material [7]:

$$
M_{s}=M_{s 0}(1-2 t / D)^{3}
$$

Hence, the more significant shrinkage of magnetic core brings about the more suppression in saturation magnetization of NPs. In a recent investigation by Kim et.al, it is estimated that by assuming thicknesses of $0.9 \mathrm{~nm}$ for disordered spin layer of maghemite nanoparticles, about $38.6 \%, 93.6 \%$ and $99.4 \%$ of spins are canted in nanoparticles with size of 12,3 and $2, \mathrm{~nm}$ respectively [75].

According to above discussion, regardless of the effects of surface coating on total weight, the main reason for decrease in magnetization of magnetite NPs is increase in surface spin disorder originated from size reduction. On the other hand, in ferrites, exchange interactions between magnetic moments in tetrahedral and octahedral sites are indirect and occur through intermediation of oxygen ions which is referred as superexchange interactions. Hence, the variations of coordination number in surface ions, due to missing of oxygen ions or the presence of defects and impurities in surface sites, cause to the more sever perturbations in superexchange interactions [10-12]. Moreover, spin canting effects can be influenced by the nature, amount/ length, composition and the thickness of organic/ inorganic surface coatings mainly because of attaching their end functional groups (such as $-\mathrm{OH},-\mathrm{COOH},-\mathrm{PO}(\mathrm{OH})_{2}, \ldots$ ) to the surface atoms of MNPs [5]. Therefore, it can be inferred that extra broken superexchange bonds originated from dextran coating of NPs are probably another reason of drastic suppression of magnetization in the dextran coated NPs with respect to the bare NPs.

It is worth to mention that the distribution of magnetic cations in tetrahedral and octahedral sites severely affects the magnetization of ferrite NPs [76]. To the best of our knowledge, no displacement of magnetic cations derived from the surface coating has been reported. 
Nevertheless, in this work, size reduction originated from in situ dextran coating of the MNPs may be cause to change the arrangement of $\mathrm{Fe}^{3+}$ and $\mathrm{Fe}^{2+}$ ions in the spinel structure of the magnetite NPs. Magnetite NPs have an inverse spinel structure of $\left(\mathrm{Fe}^{3+}\right)\left(\mathrm{Fe}^{2+} \mathrm{Fe}^{3+}\right) \mathrm{O}_{4}$ in which the first and second parenthesis are indicating tetrahedral and octahedral sites respectively $[4,76]$. A simple calculation with consideration of magnetic moment of 5 and $4 \mu_{\mathrm{B}}$ for $\mathrm{Fe}^{3+}$ and $\mathrm{Fe}^{2+}$ ions respectively, shows that net magnetic moment of each magnetite molecule is $4 \mu_{\mathrm{B}}$. However, redistribution of a fraction $(\mathrm{x})$ of magnetic cations changes magnetic structure of magnetite NPs to $\left(\mathrm{Fe}^{2+}{ }_{x} \mathrm{Fe}^{3+}{ }_{1-x}\right)\left(\mathrm{Fe}^{2+}{ }_{1-x} \mathrm{Fe}^{+3}{ }_{1+x}\right) \mathrm{O}_{4}$ [76]. This partially deviation from inverse spinel structure of magnetite NPs increases its magnetic moment to $(4+2 x) \mu_{B}$. Therefore, assuming a probable change in the distribution of magnetic cations due to size shrinkage in dextran coated NPs, the mentioned discussion on reducing the saturation magnetization is still valid. However, subtle investigation of distribution of $\mathrm{Fe}^{3+}$ and $\mathrm{Fe}^{2+}$ ions in tetrahedral and octahedral sites is beyond the scope of the present work and needs further detailed analytical and experimental studies.

\subsection{Biocompatibility study}

Fig. 10 (a) shows cell viability of L929 cells after $24 \mathrm{~h}$ exposure to the different concentrations $\left(50,100,200,300,400\right.$ and $\left.500 \mu \mathrm{g} \mathrm{ml}^{-1}\right)$ of the bare and dextran coated nanoparticles (sample B). It can be easily found that for all concentrations, cytotoxicity of the dextran coated sample is significantly less than which for the bare NPs. Although the cell viability diminishes with increase in the concentration of both samples, the concentration of $50 \mu \mathrm{g} \mathrm{ml}^{-1}$ of the sample B is still completely safe. For more investigation, the effect of cytotoxicity of the sample B on L929 cells after 24 and $48 \mathrm{~h}$ is shown in the Fig. 10 (b). As the figure shows, passing the time has a small negative effect on the cell viability of the dextran coated NPs. In addition, the cytotoxicity of the dextran coated NPs in all cell culture conditions is less than $11 \%$, indicating the more biocompatibility of the dextran coated NPs compare with the bare particles.

\section{Conclusion}

Synthesis of the bare and dextran coated magnetite NPs was successfully achieved by coprecipitation method. Characterization of samples was performed by X-ray diffraction, Fourier 
transform infrared spectroscopy, field emission scanning microscopy, transmission electron microscopy, dynamic light scattering and thermogravimetric analyses. The effect of dextran coating on magnetic properties of the bare NPs was investigated by VSM analysis. Our studies show that the dextran coated NPs have superparamagnetic property. This result can be attributed to size reduction, derived by in situ dextran coating. The experimental results also reveal that saturation magnetization of the dextran coated NPs is dramatically decreased compared with the bare NPs. The reduced magnetization of the dextran coated samples is mainly due to the presence of non-magnetic shell of dextran, since, based on thermogravimetric analysis, about sixty percent of the weight of dextran coated NPs is composed of dextran. Furthermore, surface spin disorder originated from size shrinkage is probably the other reason for significant suppression of magnetization in the dextran coated samples. Moreover, it is shown that the effect of dextran coating on magnetic properties of NPs is more distinctive when weight ratio of dextran to NPs is $2: 1$. In addition, biocompatibility studies show that surface modification of NPs dramatically decreases cytotoxicity of the bare NPs and therefore improves their efficiency as biomedical agents in theranostic applications.

\section{Acknowledgements}

This work has been supported by Arak University Research Council (AURC), Iran Nanotechnology Initiative Council (INIC), and also Iran National Science Foundation (INSF). The authors acknowledge AURC, INIC, and INSF for the financial support. They also thank Dr. M. Mahdieh for using his lab facilities and M. Nodeh Farahani for his kind help. The authors would also like to especially thank J. Ghasemi for his useful discussion and help with experimental setup. 


\section{References}

[1] D. Alcantara, L. Josephson, Chapter 11: Magnetic Nanoparticles for Application in Biomedical Sensing, Nanobiotechnology: Inorganic Nanoparticles Vs Organic Nanoparticles, 2012, pp. 269-289.

[2] A.K. Hauser, R. Mathias, K.W. Anderson, J.Z. Hilt, The effects of synthesis method on the physical and chemical properties of dextran coated iron oxide nanoparticles, Materials Chemistry and Physics, 160 (2015) 177-186.

[3] M. CHIRITA, M.L. KISS, C. SAVII, CHAPTER IV: Magnetite, Properties and Modern Biomedical Applications, New York, 2014, pp. 119-183.

[4] S. Sun, H.Zeng, D.B.Robinson, S. Raoux, P.M.Rice, S.X.Wang, G. Li, Monodisperse $\mathrm{MFe}_{2} \mathrm{O}_{4}(\mathrm{M}=\mathrm{Fe}, \mathrm{Co}, \mathrm{Mn})$ nanoparticles, Journal of the American Chemical Society, 126(1) (2004) 273-279.

[5] G. Kandasamy, D. Maity, Recent advances in superparamagnetic iron oxide nanoparticles (SPIONs) for in vitro and in vivo cancer nanotheranostics, International Journal of Pharmaceutics, 496(2) (2015) 191-218.

[6] C. $\mathrm{Xu}, \mathrm{S}$. Sun, New forms of superparamagnetic nanoparticles for biomedical applications, Advanced Drug Delivery Reviews, 65(5) (2013) 732-743.

[7] M. Muroi, R. Street, P. G. McCormick, J. Amighian, Magnetic properties of ultrafine $\mathrm{MnFe}_{2} \mathrm{O}_{4}$ powders prepared by mechanochemical processing, Physical Review B, 63(18) (2001) 184414.

[8] T. Kim, M. Shima, Reduced magnetization in magnetic oxide nanoparticles, Journal of applied physics, 101(9) (2007) 09M516.

[9] R. H. Kodama, A. E. Berkowitz, E. J. McNiff Jr, S. Foner, Surface spin disorder in ferrite nanoparticles, Journal of Applied Physics, 81(8) (1997) 5552-5557.

[10] H. Nathani, S. Gubbala, R. Misra, Magnetic behavior of nanocrystalline nickel ferrite: Part I. The effect of surface roughness, Materials Science and Engineering: B, 121(1) (2005) 126-136.

[11] H. Nathani, S. Gubbala, R. Misra, Magnetic behavior of nickel ferrite-polyethylene nanocomposites synthesized by mechanical milling process, Materials Science and Engineering: B, 111(2) (2004) 95-100. 
[12] R.H. Kodama, A.E. Berkowitz, Atomic-scale magnetic modeling of oxide nanoparticles, Physical Review B, 59(9) (1999) 6321.

[13] J. Curiale, M. Granada, H. E. Troiani, R. D. Sanchez, A. G. Leyva, P. Levy, K. Samwer, Magnetic dead layer in ferromagnetic manganite nanoparticles, Applied Physics Letters, 95(4) (2009) 043106.

[14] V. Skumryev, S. Stoyanov, Y.Zhang, G. Hadjipanayis, D. Givord, J. Nogues, Beating the superparamagnetic limit with exchange bias, Nature, 423(6942) (2003) 850-853.

[15] C. Alexiou, R.J.Schmid, R. Jurgons, M. Kremer, G. Wanner, C. Bergemann, E. Huenges, T. Nawroth, W. Arnold, F.G. Parak, Targeting cancer cells: magnetic nanoparticles as drug carriers, European Biophysics Journal, 35(5) (2006) 446-450.

[16] S. Laurent, S. Dutz, U.O. Häfeli, M. Mahmoudi, Magnetic fluid hyperthermia: Focus on superparamagnetic iron oxide nanoparticles, Advances in Colloid and Interface Science, 166(1-2) (2011) 8-23.

[17] A.G. Roca, R. Costo, A.F. Rebolledo, S. Veintemillas-Verdaguer, P. Tartaj, T. GonzalezCarreno, M.P. Morales, C.J. Serna, Progress in the preparation of magnetic nanoparticles for applications in biomedicine, Journal of Physics D: Applied Physics, 42(22) (2009) 224002.

[18] P.I. Soares, C.A. Laia, A. Carvalho, L.C. Pereira, J.T. Coutinho, I.M. Ferreira, C.M. Novo, J.P. Borges, Iron oxide nanoparticles stabilized with a bilayer of oleic acid for magnetic hyperthermia and MRI applications, Applied Surface Science, 383 (2016) 240247.

[19] Y.M. Wang, X. Cao, G.H. Liu, R.Y.Hong, Y.M. Chen, X.F. Chen, H.Z. Li, B. Xu, D.G. Wei, Synthesis of $\mathrm{Fe}_{3} \mathrm{O}_{4}$ magnetic fluid used for magnetic resonance imaging and hyperthermia, Journal of Magnetism and Magnetic Materials, 323(23) (2011) 2953-2959.

[20] Z. Li, M. Kawashita, N. Araki, M. Mitsumori, M. Hiraoka, M. Doi, Magnetite nanoparticles with high heating efficiencies for application in the hyperthermia of cancer, Materials Science and Engineering: C, 30(7) (2010) 990-996.

[21] A.K. Gupta, S. Wells, Surface-modified superparamagnetic nanoparticles for drug delivery: preparation, characterization, and cytotoxicity studies, NanoBioscience, IEEE Transactions on, 3(1) (2004) 66-73. 
[22] T. Neuberger, B. Schöpf, H. Hofmann, M. Hofmann, B. Von Rechenberg, Superparamagnetic nanoparticles for biomedical applications: Possibilities and limitations of a new drug delivery system, Journal of Magnetism and Magnetic Materials, 293(1) (2005) 483-496.

[23] M. Khalkhali, K. Rostamizadeh, S. Sadighian, F. Khoeini, M. Naghibi, M. Hamidi, The impact of polymer coatings on magnetite nanoparticles performance as MRI contrast agents: a comparative study, DARU Journal of Pharmaceutical Sciences, 23(1) (2015) 112.

[24] A. Saraswathy, S.S. Nazeer, N. Nimi, S. Arumugam, S.J. Shenoy, R.S. Jayasree, Synthesis and characterization of dextran stabilized superparamagnetic iron oxide nanoparticles for in vivo MR imaging of liver fibrosis, Carbohydrate Polymers, 101 (2014) 760-768.

[25] T.J. Chen, T.H. Cheng, C.Y. Chen, S.C. Hsu, T.L. Cheng, G.C. Liu, Y.M. Wang, Targeted Herceptin-dextran iron oxide nanoparticles for noninvasive imaging of $\mathrm{HER}_{2} /$ neu receptors using MRI, JBIC Journal of Biological Inorganic Chemistry, 14(2) (2009) 253-260.

[26] M. Huang, Z. Qiao, F. Miao, N. Jia, H. Shen, Biofunctional magnetic nanoparticles as contrast agents for magnetic resonance imaging of pancreas cancer, Microchimica Acta, 167(1-2) (2009) 27-34.

[27] R.Y. Hong, B. Feng, L.L. Chen, G.H. Liu, H.Z. Li, Y. Zheng, D.G. Wei, Synthesis, characterization and MRI application of dextran-coated Fe3O4 magnetic nanoparticles. Biochemical Engineering Journal, 42(3) (2008) 290-300.

[28] C.A.O. Zhengguo, Z.H.O.U. Siwei, L.I.U. Jihong, S.O.N.G. Xiaodong, Preparation of Superparamagnetic Dextran-coated Iron Oxide Nanoparticles used as a Novel Gene Carrier into Human Bladder Cancer Cells, The Chinese-German Journal of Clinical Oncology, 4(3) (2005) 183-186.

[29] K. Lüdtke-Buzug, S. Biederer, T.F. Sattel, T. Knopp, T.M. Buzug, Particle-Size Distribution of Dextran- and Carboxydextran-Coated Superparamagnetic Nanoparticles for Magnetic Particle Imaging, in World Congress on Medical Physics and Biomedical Engineering, September 7 - 12, 2009, Munich, Germany, (pp. 226-229), Springer Berlin Heidelberg. 
[30] H.H. Wang, Y.X.J. Wang, K.C.F. Leung, D.W. Au, S. Xuan, C.P. Chak, S.K. Lee, H. Sheng, G. Zhang, L. Qin, J.F. Griffith, Durable mesenchymal stem cell labelling by using polyhedral superparamagnetic iron oxide nanoparticles, Chemistry-A European Journal, 15(45) (2009) 12417-12425.

[31] K.S ung Kim, J.K. Park, Magnetic force-based multiplexed immunoassay using superparamagnetic nanoparticles in microfluidic channel, Lab on a Chip, 5(6) (2005) 657-664.

[32] D. Abrahamsson, K. Kriz, M. Lu, D. A. Kriz, preliminary study on DNA detection based on relative magnetic permeability measurements and histone $\mathrm{H} 1$ conjugated superparamagnetic nanoparticles as magnetic tracers, Biosensors and Bioelectronics, 19(11) (2004) 1549-1557.

[33] S.L. Easo, P.V. Mohanan, Dextran stabilized iron oxide nanoparticles: Synthesis, characterization and in vitro studies, Carbohydrate Polymers, 92(1) (2013) 726-732.

[34] R. Qiao, C. Yang, M. Gao, Superparamagnetic iron oxide nanoparticles: from preparations to in vivo MRI applications, Journal of Materials Chemistry, 19(35) (2009) 6274-6293.

[35] S. Stolnik, L. Illum, S.S. Davis, Long circulating microparticulate drug carriers, Advanced Drug Delivery Reviews, 64 (2012) 290-301.

[36] V.M. Khot, A.B. Salunkhe, N.D. Thorat, R.S. Ningthoujam, S.H. Pawar, Induction heating studies of dextran coated $\mathrm{MgFe}_{2} \mathrm{O}_{4}$ nanoparticles for magnetic hyperthermia, Dalton Transactions, 42(4) (2013) 1249-1258.

[37] H.T. Chan, Y.Y. Do, P.L. Huang, P.L. Chien, T.S. Chan, R.S. Liu, C.Y. Huang, S.Y. Yang, H.E. Horng, Preparation and properties of bio-compatible magnetic Fe3O4 nanoparticles, Journal of Magnetism and Magnetic Materials, 304(1) (2006) e415-e417.

[38] R.S. Molday, D. Mackenzie, Immunospecific ferromagnetic iron-dextran reagents for the labeling and magnetic separation of cells, Journal of immunological methods, 52(3) (1982) 353-367.

[39] M.I. Papisov, A. Bogdanov, B. Schaffer, N. Nossiff, T. Shen, R. Weissleder, T.J. Brady, Colloidal magnetic resonance contrast agents: effect of particle surface on biodistribution, Journal of Magnetism and Magnetic Materials, 122(1-3) (1993) 383-386. 
[40] L.M. Lacava, V.A.P. Garcia, S. Kückelhaus, R.B. Azevedo, N. Sadeghiani, N. Buske, P.C. Morais, Z.G.M. Lacava, Long-term retention of dextran-coated magnetite nanoparticles in the liver and spleen, Journal of Magnetism and Magnetic Materials, 272 (2004) 2434-2435.

[41] M.M. Molina, A.B. Seabra, M.G. de Oliveira, R. Itri, P.S. Haddad, Nitric oxide donor superparamagnetic iron oxide nanoparticles, Materials Science and Engineering: C, 33(2) (2013) 746-751.

[42] E.A. Osborne, T.M. Atkins, D.A. Gilbert, S.M. Kauzlarich, K. Liu, A.Y. Louie, Rapid microwave-assisted synthesis of dextran-coated iron oxide nanoparticles for magnetic resonance imaging, Nanotechnology, 23(21) (2012) 215602.

[43] E.H. Kim, H.S. Lee, B.K. Kwak, B.K. Kim, Synthesis of ferrofluid with magnetic nanoparticles by sonochemical method for MRI contrast agent, Journal of Magnetism and Magnetic Materials, 289 (2005) 328-330.

[44] Y. Hou, J. Yu, S. Gao, Solvothermal reduction synthesis and characterization of superparamagnetic magnetite nanoparticles, Journal of Materials Chemistry, 13(8) (2003) 1983-1987.

[45] L. Xiao, J. Li, D.F. Brougham, E.K. Fox, N. Feliu, A. Bushmelev, A. Schmidt, N. Mertens, F. Kiessling, M. Valldor, B. Fadeel, Water-Soluble Superparamagnetic Magnetite Nanoparticles with Biocompatible Coating for Enhanced Magnetic Resonance Imaging, ACS Nano, 5(8) (2011) 6315-6324.

[46] R. Ahmadi, H. Hosseini, An investigation on the optimum conditions of synthesizing a magnetite based ferrofluid as MRI contrast agent using Taguchi method, Materials Science-Poland, 31(2) (2013) 253-258.

[47] T. Hosono, H. Takahashi, A. Fujita, R.J.Joseyphus, K.Tohji, B. Jeyadevan, Synthesis of magnetite nanoparticles for AC magnetic heating, Journal of Magnetism and Magnetic Materials, 321(19) (2009) 3019-3023.

[48] N. Sattarahmady, T. Zare, A.R. Mehdizadeh, N. Azarpira, M. Heidari, M.Lotfi, H. Heli, Dextrin-coated zinc substituted cobalt-ferrite nanoparticles as an MRI contrast agent: In vitro and in vivo imaging studies, Colloids and Surfaces B: Biointerfaces, 129 (2015) 1520. 
[49] J.P. Chen, C.M. Sorensen, K.J. Klabunde, G.C. Hadjipanayis, E. Devlin, A. Kostikas, Size-dependent magnetic properties of $\mathrm{MnFe}_{2} \mathrm{O}_{4}$ fine particles synthesized by coprecipitation, Physical Review B, 54(13) (1996) 9288-9296.

[50] S. Gyergyek, M. Drofenik, D. Makovec, Oleic-acid-coated $\mathrm{CoFe}_{2} \mathrm{O}_{4}$ nanoparticles synthesized by co-precipitation and hydrothermal synthesis, Materials Chemistry and Physics, 133(1) (2012) 515-522.

[51] B. Jeyadevan, C.N. Chinnasamy, K.Shinoda, K.Tohji, H. Oka, Mn-Zn ferrite with higher magnetization for temperature sensitive magnetic fluid, Journal of applied physics, 93(10) (2003) 8450-8452.

[52] A.K. Gupta, M. Gupta, Synthesis and surface engineering of iron oxide nanoparticles for biomedical applications, Biomaterials, 26(18) (2005) 3995-4021.

[53] D.K. Kim, Y.Zhang, W.Voit, K.V.Rao, M. Muhammed, Synthesis and characterization of surfactant-coated superparamagnetic monodispersed iron oxide nanoparticles, Journal of Magnetism and Magnetic Materials, 225(1) (2001) 30-36.

[54] J.P. Jolivet, C.Chanéac, P.Prene, L.Vayssieres, E. Tronc, Wet chemistry of spinel iron oxide particles, Le Journal de Physique IV, 7(C1) (1997) C1-573-C1-576.

[55] L. Vayssieres, C. Chanéac, E.Tronc, J.P. Jolivet, Size Tailoring of Magnetite Particles Formed by Aqueous Precipitation: An Example of Thermodynamic Stability of Nanometric Oxide Particles, Journal of Colloid and Interface Science, 205(2) (1998) 205212.

[56] G. Liu, R.Y. Hong, L. Guo, Y.G. Li, H.Z. Li, Preparation, characterization and MRI application of carboxymethyl dextran coated magnetic nanoparticles, Applied Surface Science, 257(15) (2011) 6711-6717.

[57] M. C. Bautista, O. Bomati-Miguel, M. del Puerto Morales, C. J. Serna and S. Veintemillas-Verdaguer, Surface characterisation of dextran-coated iron oxide nanoparticles prepared by laser pyrolysis and coprecipitation, Journal of magnetism and magnetic materials, 293(1) (2005) 20-27.

[58] M. Latorre-Esteves, A. Cortés, M. Torres-Lugo, and C. Rinaldi, Synthesis and characterization of carboxymethyl dextran-coated $\mathrm{Mn} / \mathrm{Zn}$ ferrite for biomedical applications, Journal of Magnetism and Magnetic Materials, 321(19) (2009) 3061-3066. 
[59] I. Sharifi, H. Shokrollahi, and S. Amiri, Ferrite-based magnetic nanofluids used in hyperthermia applications, Journal of Magnetism and Magnetic Materials, 324(6) (2012) 903-915.

[60] O. Ciftja, Lamellar-like structures in ferrofluids placed in strong magnetic fields, Solid State Communications, 149(13) (2009) 532-536.

[61] J. Li, Y. Huang, X. Liu, Y. Lin, L. Bai, and Q. Li, Effect of aggregates on the magnetization property of ferrofluids: A model of gaslike compression, Science and Technology of Advanced Materials, 8(6) (2007) 448-454.

[62] O. Carp, L. Patron, D. Culita, P. Budrugeac, M. Feder, L. Diamandescu, Thermal analysis of two types of dextran-coated magnetite, Journal of Thermal Analysis and Calorimetry, 101(1) (2010) 181-187.

[63] E.C. Stoner, E. Wohlfarth, A mechanism of magnetic hysteresis in heterogeneous alloys, Philosophical Transactions of the Royal Society of London, Series A. Mathematical and Physical Sciences, 240(826) (1948) 599-642.

[64] M.A. López-Quintela, J. Rivas, Nanoscale magnetic particles: synthesis, structure and dynamics. Current Opinion in Colloid \& Interface Science, 1(6) (1996) 806-819.

[65] A. Ahlawat, V.G. Sathe, V.R. Reddy, A. Gupta, Mossbauer, Raman and X-ray diffraction studies of superparamagnetic $\mathrm{NiFe}_{2} \mathrm{O}_{4}$ nanoparticles prepared by sol-gel autocombustion method, Journal of Magnetism and Magnetic Materials, 323(15) (2011) 2049-2054.

[66] A.E. Deatsch, B.A. Evans, Heating efficiency in magnetic nanoparticle hyperthermia, Journal of Magnetism and Magnetic Materials, 354 (2014) 163-172.

[67] M. Knobel, W.C. Nunes, L.M. Socolovsky, E. De Biasi, J.M. Vargas, J.C. Denardin, Superparamagnetism and other magnetic features in granular materials: a review on ideal and real systems, Journal of Nanoscience and Nanotechnology, 8(6) (2008) 2836-2857.

[68] C. Mee, E. Daniel 2nd, Magnetic Recording Technology, McGraw-Hill, New York, 1996.

[69] R. Hergt, W. Andra, C.G. d'Ambly, I. Hilger, W.A. Kaiser, U. Richter, H.G. Schmidt, Physical limits of hyperthermia using magnetite fine particles, IEEE Transactions on Magnetics, 34(5) (1998) 3745-3754.

[70] R.E. Rosensweig, Heating magnetic fluid with alternating magnetic field, Journal of Magnetism and Magnetic Materials, 25 (2002) 370-374. 
[71] B. Jeyadevan, Present status and prospects of magnetite nanoparticles-based hyperthermia, Journal of the Ceramic Society of Japan, 118(1378) (2010) 391-401.

[72] W. Wu, Q. He, C. Jiang, Magnetic Iron Oxide Nanoparticles: Synthesis and Surface Functionalization Strategies, Nanoscale Research Letters, 3(11) (2008) 397-415.

[73] R.H. Kodama, A.E. Berkowitz, E.J. McNiff Jr, S. Foner, Surface spin disorder in $\mathrm{NiFe}_{2} \mathrm{O}_{4}$ nanoparticles, Physical Review Letters, 77(2) (1996) 394.

[74] R.H. Kodama, Magnetic nanoparticles, Journal of Magnetism and Magnetic Materials, 200(1) (1999) 359-372.

[75] B.H. Kim, N. Lee, H. Kim, K. An, Y.I. Park, Y. Choi, K. Shin, Y.Lee, S.G. Kwon, H.B. Na, J.G. Park, Large-Scale Synthesis of Uniform and Extremely Small-Sized Iron Oxide Nanoparticles for High-Resolution $\mathrm{T}_{1}$ Magnetic Resonance Imaging Contrast Agents, Journal of the American Chemical Society, 133(32) (2011) 12624-12631.

[76] B.D. Cullity, C.D. Graham, Introduction to magnetic materials, John Wiley \& Sons, 2011 


\section{Table captions:}

Table 1

The magnetic parameters (coercivity $\left(H_{c}\right)$, saturation magnetization $\left(M_{s}\right)$ and remanence $\left(M_{r}\right)$ ) of the bare and dextran coated NPs.

\section{Figure captions:}

Fig. 1. Schematic representation of preparing steps of the bare and dextran coated NPs.

Fig. 2. FESEM images of the bare NPs ((a) and (b)) as well as the samples A and B ((c) and (d) respectively).

Fig. 3. Stability of (a) the bare NPs: (Right) immediately after preparing, (Left) after $1 \mathrm{~h}$ and (b) the dextran coated NPs (sample B): (Right) immediately after preparing, (Left) after several months.

Fig. 4. TEM images of (a) the bare NPs and (b) the NPs of samples B.

Fig. 5. Hydrodynamic size distribution of the dextran coated NPS of sample B.

Fig. 6. X-ray diffraction patterns of (a) pure dextran and (b) the bare and dextran coated NPs.

Fig. 7. FT-IR spectra of the bare and dextran coated NPs as well as pure dextran.

Fig. 8. TGA curves of the pure dextran as well as the bare and dextran coated NPs.

Fig. 9. Magnetization curves of the bare and dextran coated magnetite NPs at room temperature.

The inset shows a magnified view of the central region of loops.

Fig. 10. Cytotoxicity of nanoparticles at different concentrations: (a) the bare and dextran coated NPs (sample B) after $24 \mathrm{~h}$, (b) the dextran coated NPs (sample B) after 24 and $48 \mathrm{~h}$. 
Table 1 . The magnetic parameters (coercivity $\left(H_{c}\right)$, saturation magnetization $\left(M_{S}\right)$ and remanence $\left.\left(M_{r}\right)\right)$ of the bare and dextran coated NPs.

\begin{tabular}{lccc}
\hline & $\boldsymbol{H}_{\boldsymbol{c}}(\mathrm{Oe})$ & $\boldsymbol{M}_{\boldsymbol{s}}(\mathrm{emu} / \mathrm{g})$ & $\boldsymbol{M}_{\boldsymbol{r}}(\mathrm{emu} / \mathrm{g})$ \\
\hline Bare NPs & 6.88 & 58.91 & 0.820 \\
Dextran coated NPs (A) & 0.83 & 12.10 & 0.011 \\
Dextran coated NPs (B) & 0.09 & 7.26 & 0.008 \\
\hline
\end{tabular}




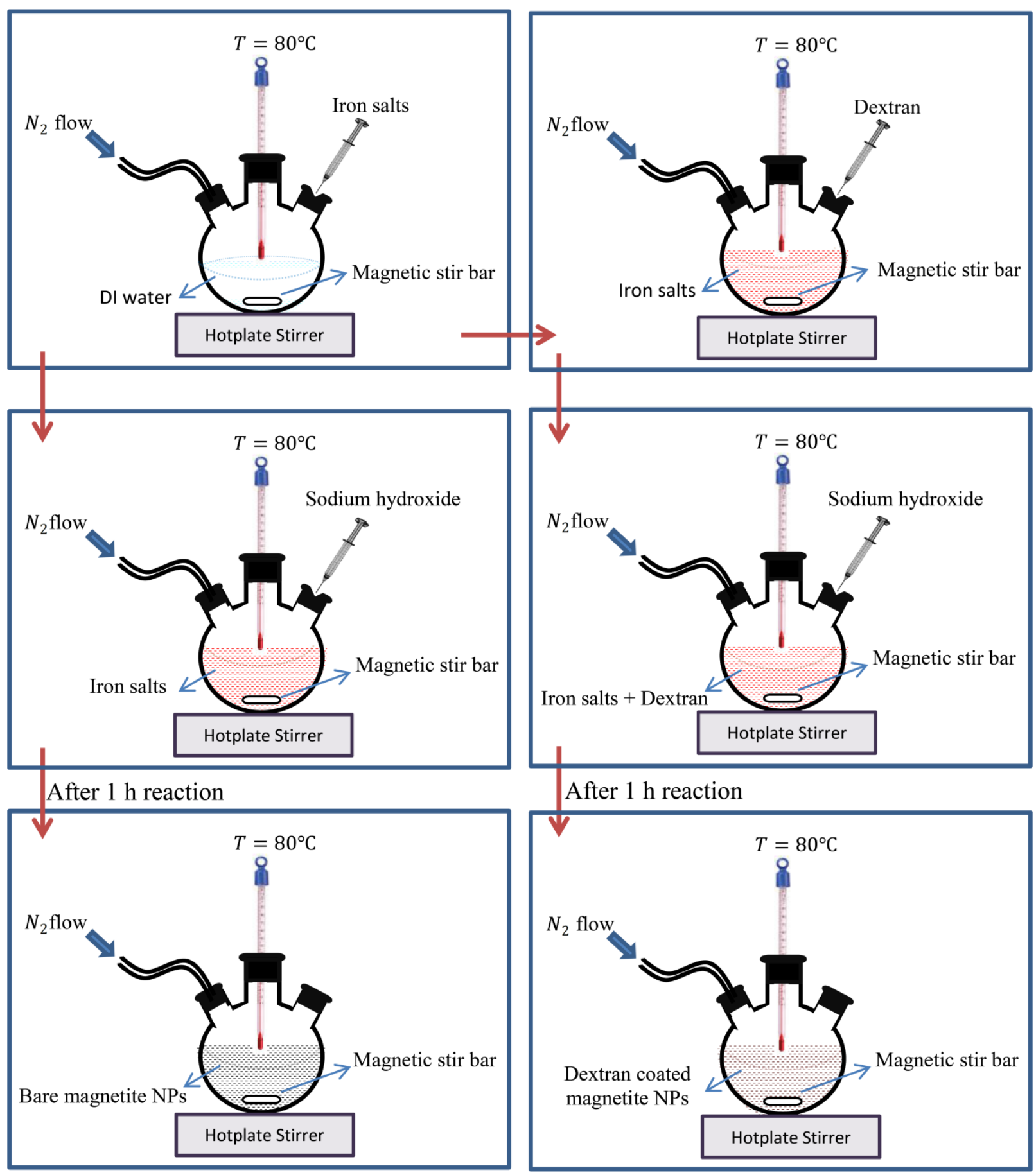

Fig. 1. Schematic representation of preparing steps of the bare and dextran coated NPs. 



Fig. 2. FESEM images of the bare NPs ((a) and (b)) as well as the samples A and B ((c) and (d) respectively). 

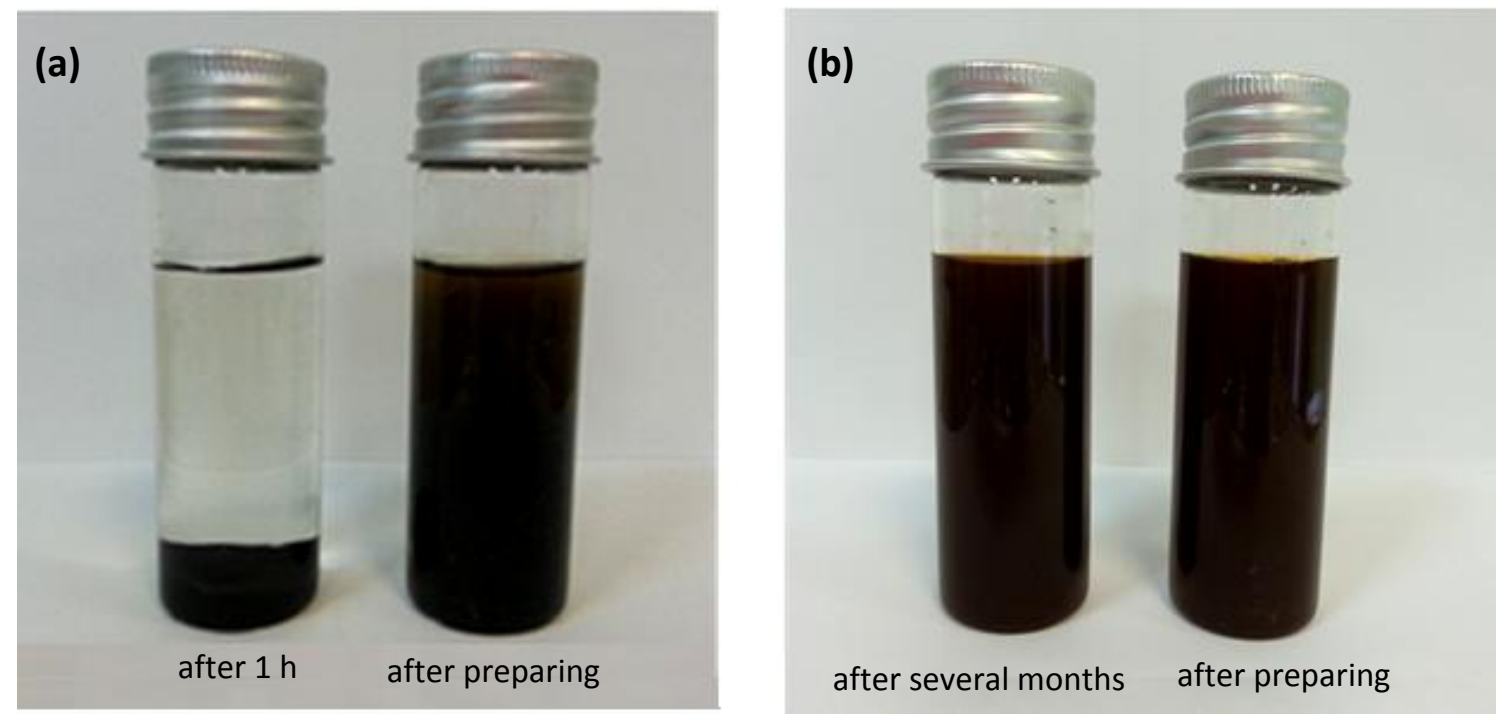

Fig. 3. Stability of (a) the bare NPs: (Right) immediately after preparing, (Left) after 1h and (b) the dextran coated NPs (sample B): (Right) immediately after preparing, (Left) after several months. 

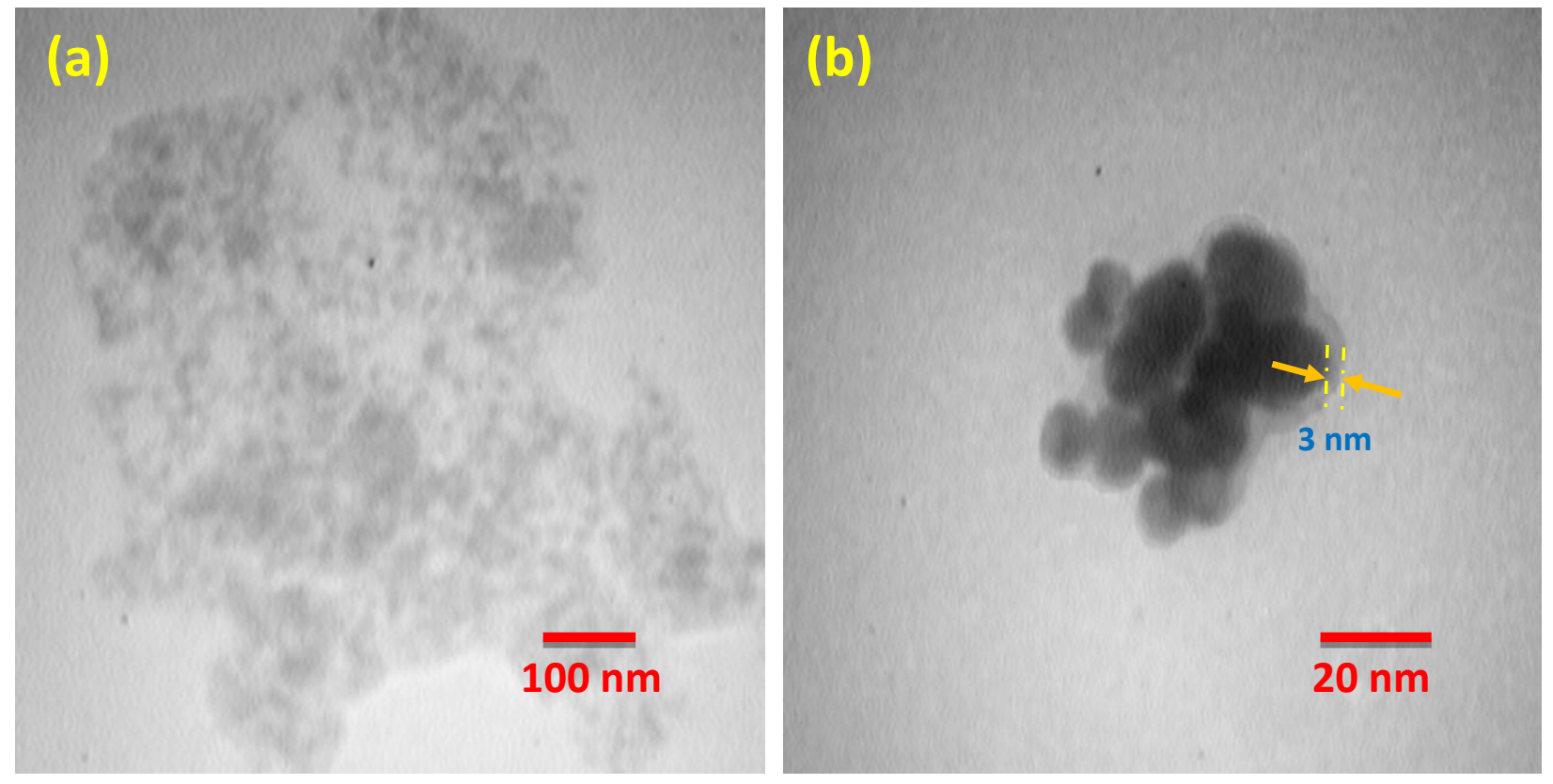

Fig. 4. TEM images of (a) the bare NPs and (b) the NPs of samples B. 


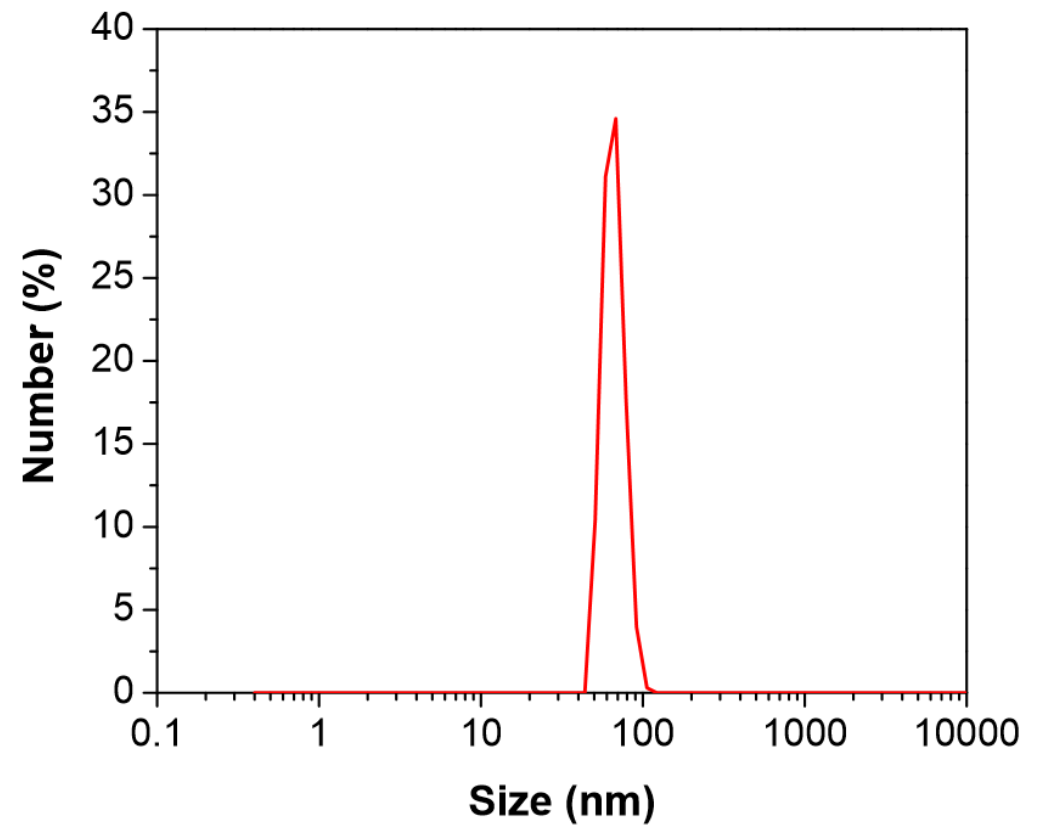

Fig. 5. Hydrodynamic size distribution of the dextran coated NPS of sample B. 

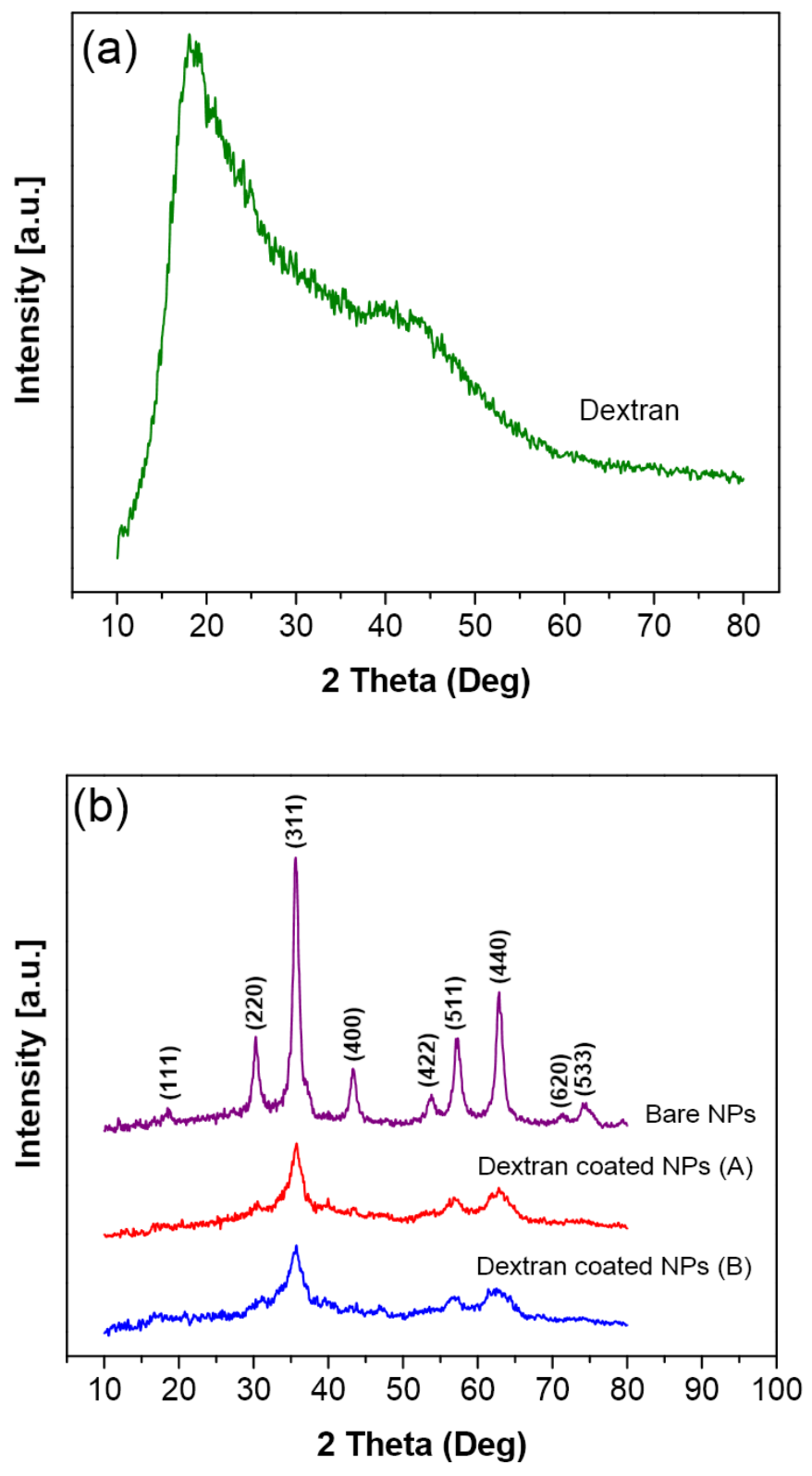

Fig. 6. X-ray diffraction patterns of (a) pure dextran and (b) the bare and dextran coated NPs. 


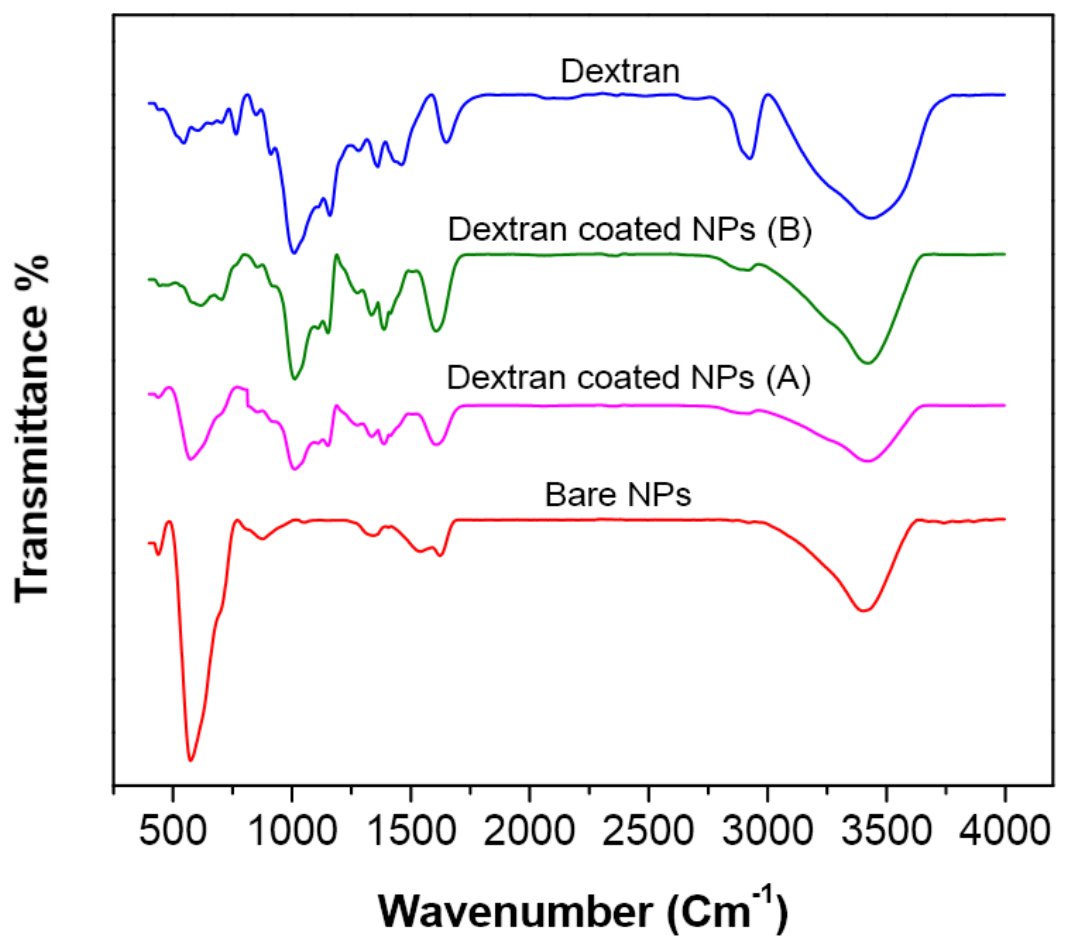

Fig. 7. FT-IR spectra of the bare and dextran coated NPs as well as pure dextran. 


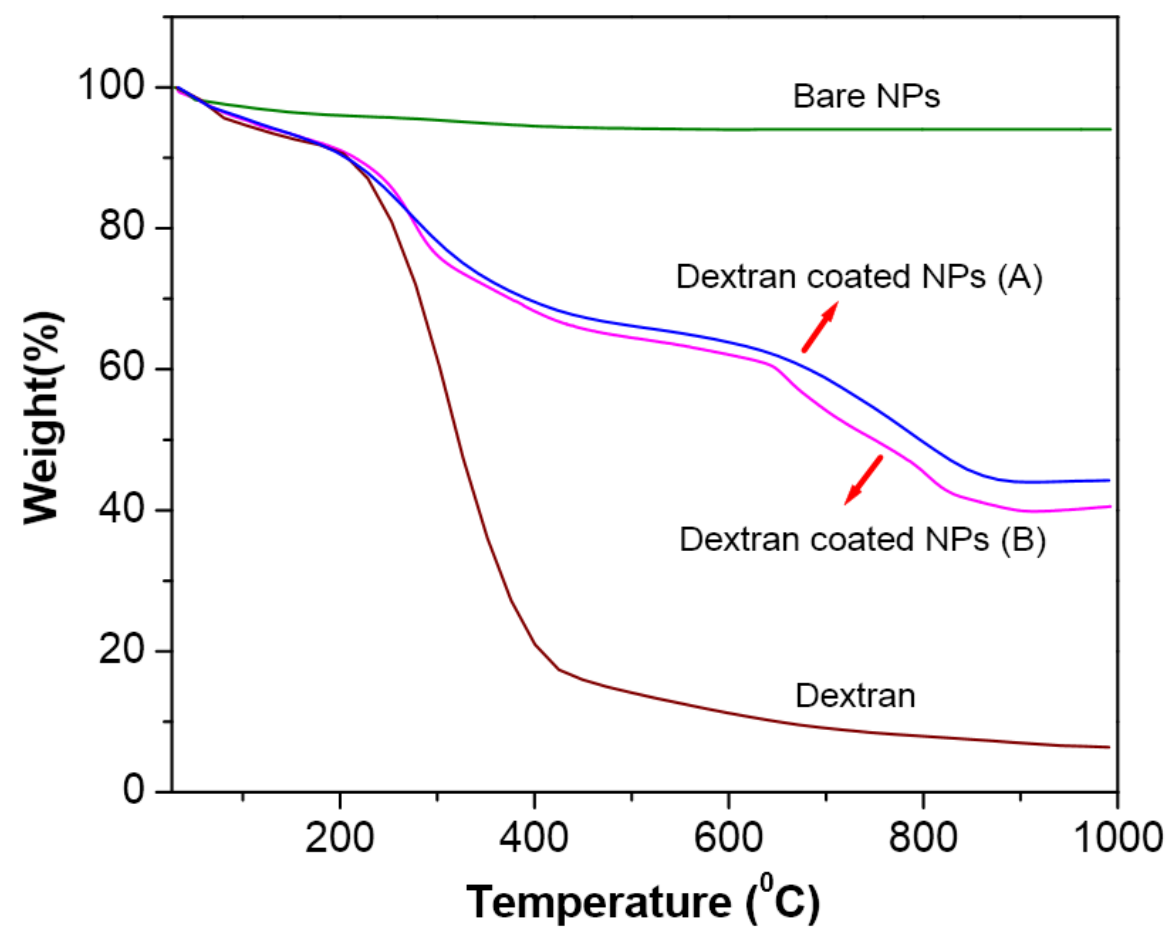

Fig. 8. TGA curves of the pure dextran as well as the bare and dextran coated NPs. 


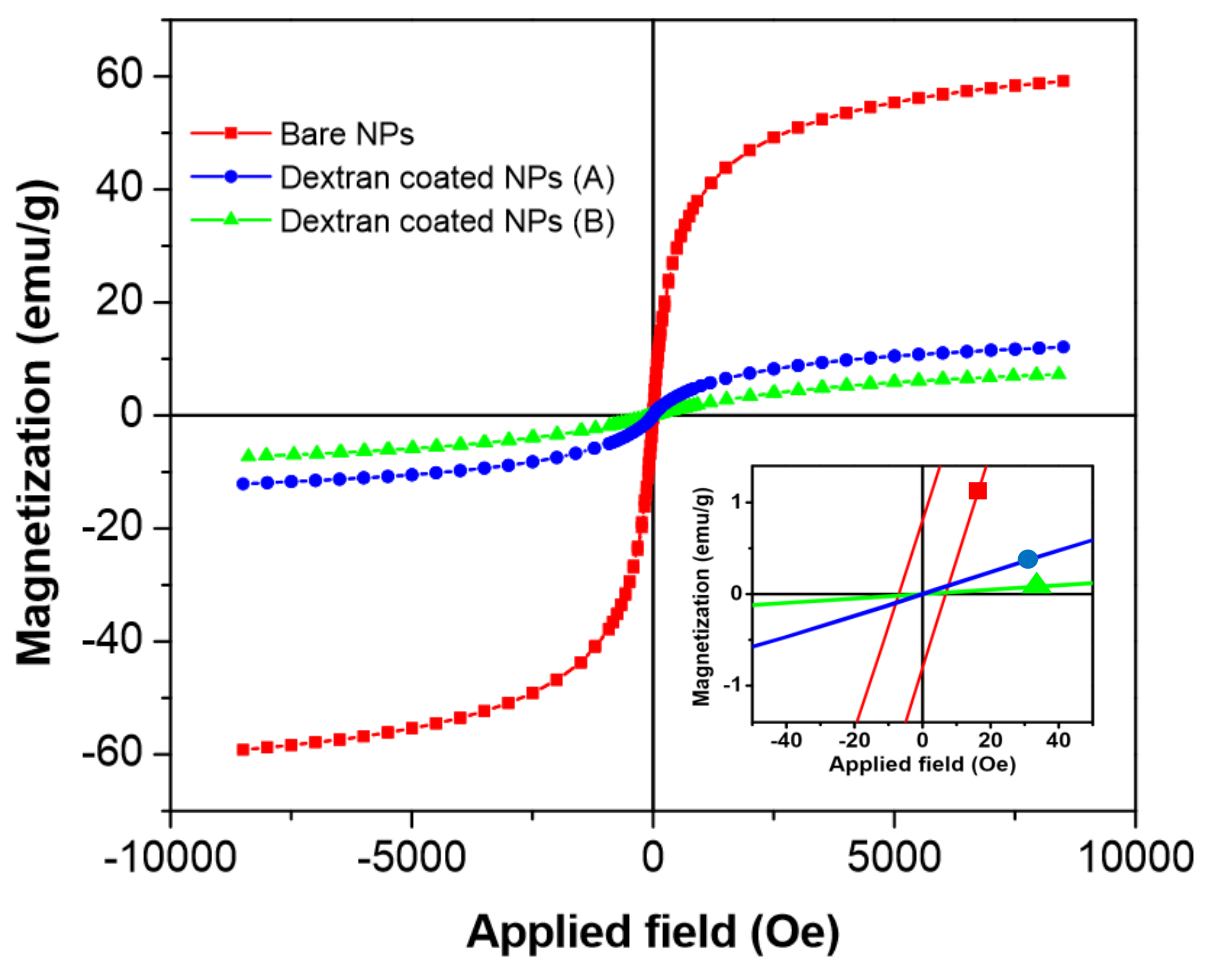

Fig. 9. Magnetization curves of the bare and dextran coated magnetite NPs at room temperature. The inset shows a magnified view of the central region of loops. 

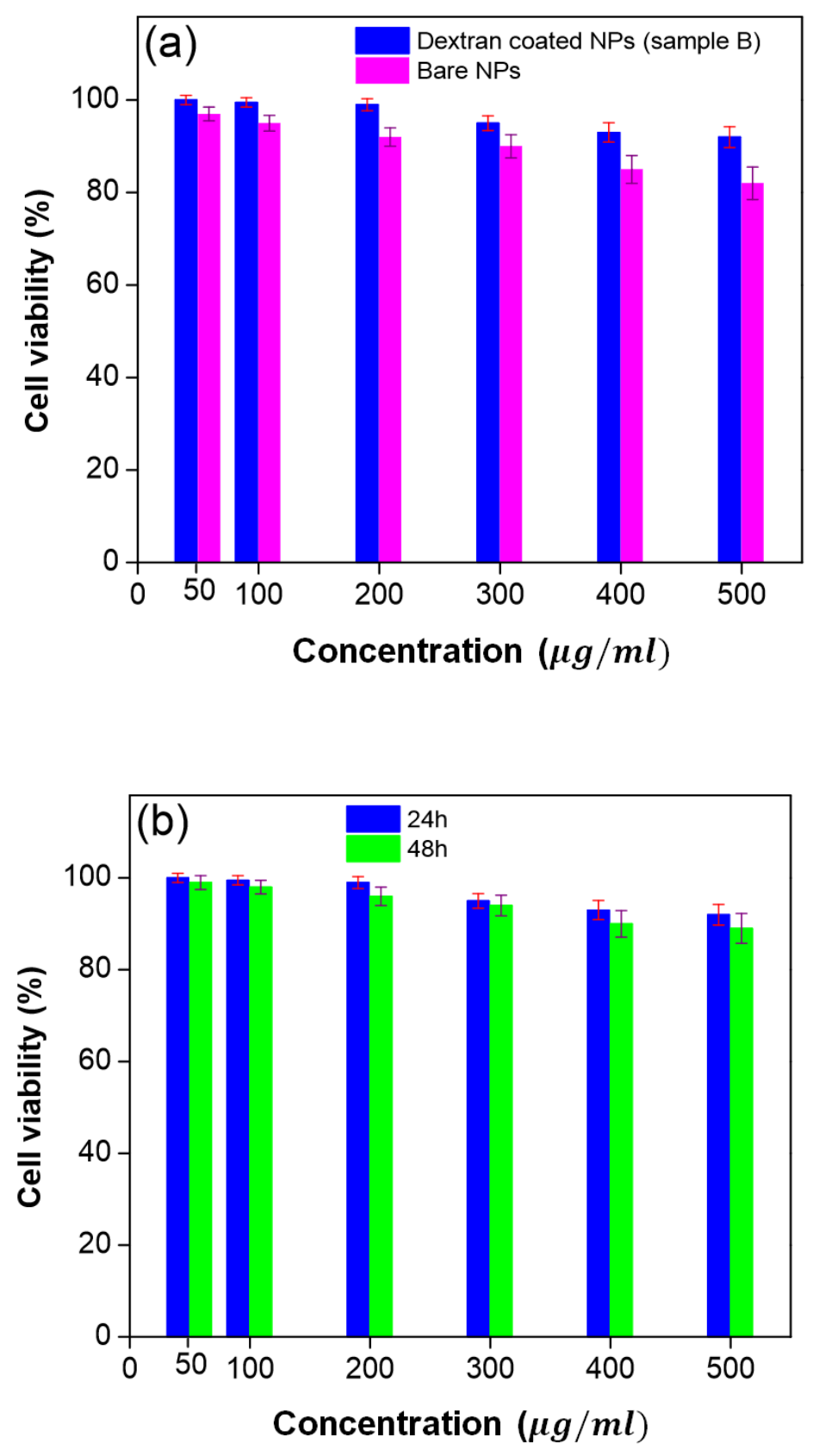

Fig. 10. Cytotoxicity of nanoparticles at different concentrations: (a) the bare and dextran coated NPs (sample B) after $24 \mathrm{~h}$, (b) the dextran coated NPs (sample B) after 24 and $48 \mathrm{~h}$. 

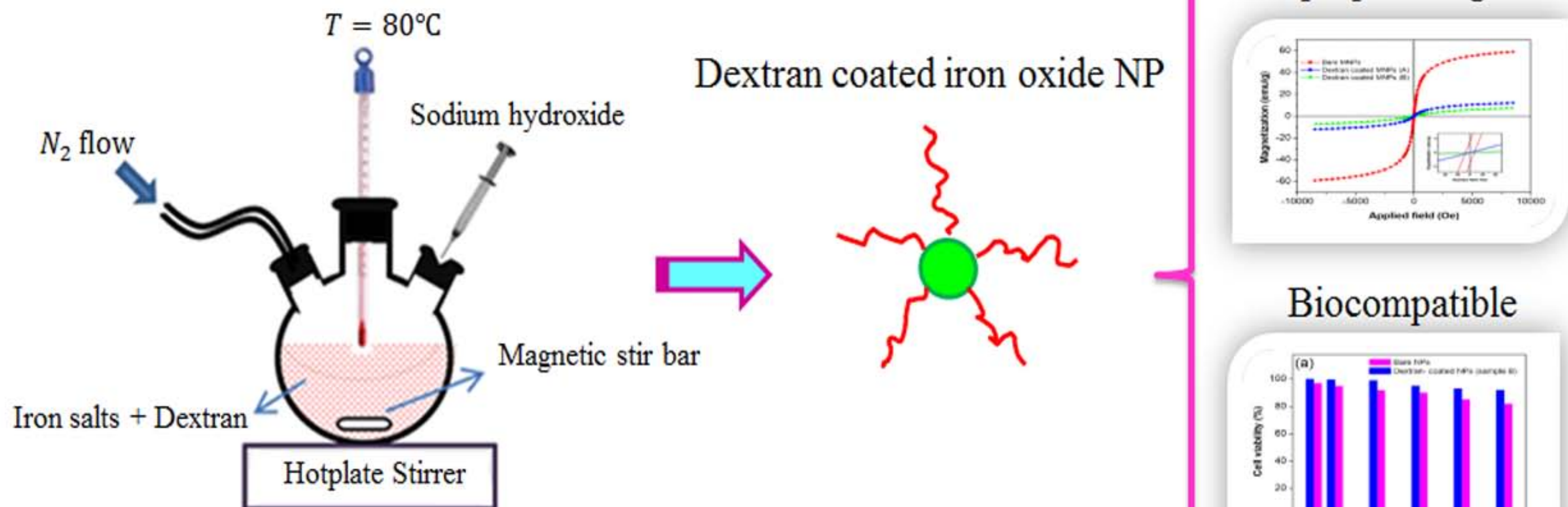

Biocompatible

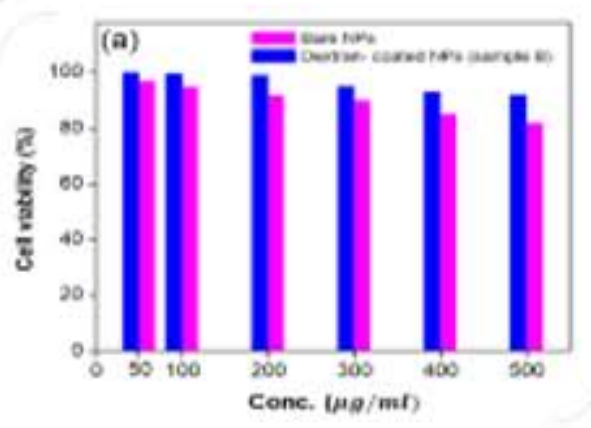

